\title{
Expression of pMGN(-4k)LacZ-neo Gene Introduced into Embryonic Stem Cells In Vitro
}

\author{
Makoto Osonoi*, Yuka Mimaki, Kazuei Ito, Jutaro Takahashi and \\ Yasuhisa Yasuda
}

Laboratory of Animal Breeding and Reproduction, Faculty of Agriculture, Iwate University, Morioka, Iwate 020-8550, Japan

\begin{abstract}
We attempted to produce a muscle-specific cell marker, which would be useful for examining myogenesis, by introducing pMGN(-4k)LacZ-neo gene into ESD3 cells, and expression of the transgene was analyzed in embryoid bodies. The gene expression analysis of embryoid bodies indicated that transfected cells did not express LacZ gene in an undifferentiated state and that the number of cells with LacZ expression increased progressively. In DMSO treated embryoid bodies, the number of cells expressing LacZ gene tended to increase more rapidly than that of untreated ones. The results suggested that the DMSO may induce mesodermal differentiation of ES cells and that the sublines obtained might be useful as muscle-specific cell markers since they might express lacZ gene at the time of myogenin expression.

Key words: Embryonic stem (ES) cells, Gene expression, Myogenin gene regulatory region, LacZ reporter gene, Embryoid bodies.
\end{abstract}

Cell markers used for histochemical analysis of chimeric mice are desired to express in all cell types at all developmental stages and be easily detectable in all tissues at a cellular level. However there are few reports of useful endogenous markers. In 1988, Kusakabe et al. [1] reported the $\mathrm{C} 3 \mathrm{H}$ strain specific antigen which may be an ideal histological marker, and in 1996, Kitani et al. [2] established embryonic stem (ES) cell lines derived from $\mathrm{C} 3 \mathrm{H} / \mathrm{He}$ mice. These cell lines are probably useful for analysis of chimeric mice at a cellular level. While groping endogenous markers, useful cell markers were produced by introducing an exogenous transgene into pluripotential cells. Hanaoka et al. [3]

Received: October 18, 1998

Accepted: December 30, 1998

*To whom correspondence should be addressed. produced cell markers by transfection of the bacterial chloramphenicol acetyltransferase gene driven by the human elongation factor $1 \alpha$ promoter into embryonal carcinoma (EC) cells and ES cells. Kadokawa et al. [4] reported production of a cell marker using $\beta$-galactosidase (LacZ) gene driven by RSV enhancer and chicken $\beta$-actin promoter. These cell markers fulfill the desirable nature as described for tissue-non-specific cell markers. However, in order to examine development or differentiation of specific tissue or organ, tissue-specific cell markers would be useful. Furthermore, such markers could also be used for the analysis of diverse functions of a gene and the studies of transplantation of cells or tissues into animals.

Myogenin is one of the myogenic regulatory genes and is expressed during embryogenesis as early as the first somite/myotome formation. Its transcripts are detected at 8.5 days of gestation in the myotome compartment of the somites, and are expressed at high levels by 9.25 days post coitum (dpc) [5]. After that, expression of myogenin extends to trunk and limb buds. Therefore, myogenin is crucial to early myogenesis and it is almost specific to skeletal muscle. The regulatory region of a gene such as the myogenin gene, which is expressed during differentiation of a specific tissue, and which continues to be expressed during morphogenesis, would be useful for producing a tissue-specific cell marker for analysis of morphogenesis and diverse functions of a gene. Fujisawa et al. [6] and Kim et al. [7] reported expression of LacZ gene driven by the myogenin regulatory region in transgenic mouse embryos. These reports indicated the usefulness of myogenin-LacZ gene as a marker to study myogenin gene expression.

In this study, we attempted to produce a tissue-specific cell marker by transfection of a foreign gene into ES 
cells. In order to produce a muscle-specific cell marker, we used pMGN(-4k)LacZ gene as a transgene and the ESD3 cell line. We report here the transfection of transgene into ES cells, and the expression of LacZ reporter gene in embryoid bodies during early differentiation.

\section{Materials and Methods}

\section{Preparation of transgene}

In this study, pMGN(-4k)LacZ gene (supplied by the National Institute of Neuroscience) [6, 8], which consists of LacZ gene driven by the myogenin gene regulatory region, was used as a transgene. In order to select transfected cells in vitro, a neomycin resistant gene (Sal I-Xho I fragment of pMC1neopolyA (STRATAGENE)) was fused at the Sal I site of pMGN(4k)LacZ gene (Fig. 1).

In order to introduce the transgene into ES cells, pMGN(-4k)LacZ-neo gene was linearized by digestion with Sal I, after fusion and replication. The linearized transgene was dissolved in HEPES buffered saline at a concentration of $60 \mathrm{nM}$.

\section{ES cells}

The ESD3 cell line which was established from 129/ Sv strain mice by Doetchman et al. [9], and which was used for inducing differentiation into musculature by Dinsmore et al. [10], was used in this study. This cell line was supplied by Tsukuba University at 14 passages, and cells up to 20 passages were used for transfection. The cells were maintained on feeder layers, which were $\mathrm{BALB} / \mathrm{c},(\mathrm{BALB} / \mathrm{C} \times \mathrm{C} 57 \mathrm{BL} / 6) \mathrm{F} 1$ or $(\mathrm{BALB} / \mathrm{C} \times \mathrm{C} 57 \mathrm{BL} /$ 6)F2 fetal fibroblasts cultured in Dulbecco's modified Eagle medium (DMEM) supplemented with $10 \%$ newborn calf serum and treated with mitomycin C (SIGMA or KYOWA), in high-glucose DMEM supplemented with $10 \%$ fetal calf serum, 2-mercaptoethanol, non-essential amino acids solution and nuclosides (see [11] in detail, it is referred to as ESM in the present report.). To transfect with the transgene, ESD3 cells were prepared in HEPES buffered saline at a concentration of $2.0 \times$ $10^{7}$ cells $/ \mathrm{ml}$, after removing feeder cells.

\section{Transfection, selection and expansion of sublines}

In order to transfect ESD3 cells with pMGN(-4k)LacZneo gene, $0.4 \mathrm{ml}$ of cell suspension and $0.1 \mathrm{ml}$ of solution of transgene were mixed and transferred into a cuvette. ESD3 cells were electroporated with the transgene under conditions of $250 \mathrm{~V}, 960 \mu \mathrm{F}$ and resistance infinity (time constant was 55-57 msec when electroporation was performed with these conditions). Electroporation

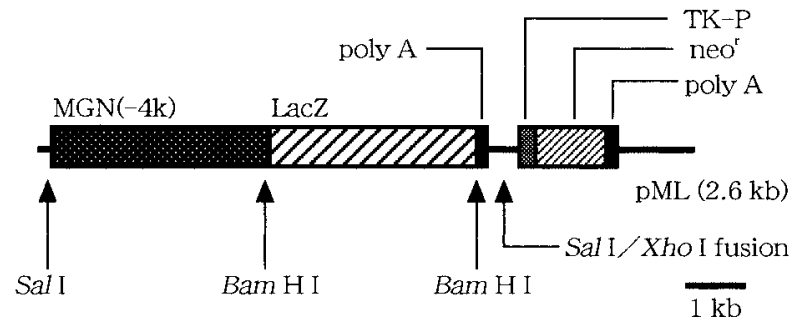

Fig. 1. The structure of pMGN(-4k)LacZ-neo gene, i.e. pMGN(-4k)LacZ gene which consists of LacZ gene driven by myogenin gene regulatory region, fused with neomycin resistant gene (Sal I-Xho I fragment from pMC1neoPolyA) into Sal I site of pMGN($4 \mathrm{k})$ LacZ gene. The LacZ gene (Bam HI-Bam HI fragment from pMGN(-4k)LacZ gene) was used as a probe for Southern hybridization. MGN(-4k): myogenin gene regulatory region, LacZ: $\beta$-galactosidase gene, TK-P: thymidine kinase promoter, neor: neomycin resistant gene.

was carried out with 2 cuvettes under the same conditions. After electroporation, the suspension was allowed to recover from electric damage for about $10 \mathrm{~min}$ at room temperature. Thirty milliliters of ESM supplemented with $1000 \mathrm{U} / \mathrm{ml}$ leukemia inhibitory factor (LIF; ESGRO, GIBCO) were added to the cuvette content, and transferred to 3 gelatinized $100 \mathrm{~mm}$ tissue culture dishes. The medium of these dishes was changed to fresh ESM $(\mathrm{LIF}+)$ after 24 hours. After 48 hours, the medium was changed to ESM (LIF+) supplemented with $200 \mu \mathrm{g} / \mathrm{ml}$ G418 to select transfected cells. Selection culture was performed for 10 days, changing the medium daily.

To determine the survival rate of cells after electroporation, three gelatinized $60 \mathrm{~mm}$ tissue culture dishes were also prepared for ESD3 control cells and transfected cells after about 10 min of transfection. These cells were cultured for 7 days changing the medium without G418 daily. After 7 days, colonies were counted and the survival rate was calculated.

After selection for 10 days with G418, five undifferentiated colonies were picked up from each dish, transferred into gelatinized $35 \mathrm{~mm}$ tissue culture dishes after disaggregation, and cultured in ESM (LIF+) for 23 days. After first passage, these sublines were maintained in $35 \mathrm{~mm}$ or $60 \mathrm{~mm}$ dishes by ordinary procedure using feeder layers. All 30 sublines were expanded, and prepared for confirming of gene integration by Southern hybridization.

\section{Southern hybridization}

All sublines were cultured in gelatinized $60 \mathrm{~mm}$ tis- 
sue culture dishes for 2-3 days, and used for extraction of genomic DNA. Cells from each subline were recovered by scraping each dish, and suspended in 0.5-0.7 $\mathrm{ml}$ of lysis buffer (10 mM Tris- $\mathrm{HCl}$ ( $\mathrm{pH} 8.0), 150 \mathrm{mM}$ $\mathrm{NaCl}, 10 \mathrm{mM}$ ethylenediaminetetraacetic acid (EDTA)) in $1.5 \mathrm{ml}$ tubes after washing with phosphate buffered saline (PBS). Each suspention was supplemented with $2 \mu \mathrm{l}$ of $20 \%$ sodium dodecyl sulfate (SDS) and $2 \mu \mathrm{l}$ of $10 \mathrm{mg} / \mathrm{ml}$ proteinase $\mathrm{K}$, and incubated overnight at $55^{\circ} \mathrm{C}$. The genomic DNA of each subline was purified by phenol extraction, and extracted by isopropyl alcohol.

Genomic DNA of each subline was digested with $B a m \mathrm{H} \mathrm{I}$ and electrophoresed in 1\% agarose gels and transfered to a nylon membrane. A probe for Southern hybridization was prepared from the LacZ gene (BamH I/BamH I fragment from pMGN(-4k)LacZ gene purified by $1 \%$ agarose gel electrophoresis, see Fig. 1) by the random primer method. Southern hybridization of the genomic DNA was carried out overnight at $62^{\circ} \mathrm{C}$ in a hybridization solution (0.25 $\mathrm{M} \mathrm{NaHPO}_{4}, 1 \mathrm{mM}$ EDTA, $7 \%$ SDS) with the probe after pre-hybridization ( 1 hour, $62^{\circ} \mathrm{C}$, in a hybridization solution without the probe). The membranes were exposed to X-ray films for about 24 hours at $-80^{\circ} \mathrm{C}$, and developed.

\section{Karyotype analysis}

Karyotype analysis of the ESD3 parent cell line and the G418-resistant sublines which contained LacZ gene was carried out by standard procedure based on the method described by Robertson [11]. Cultures were treated with colcemid (final concentration $0.02 \mu \mathrm{g} / \mathrm{ml}$ ) for about 1 hour. After removal of feeder cells, hypotonic treatment was performed with $0.56 \% \mathrm{KCl}$ solution for 10-20 $\mathrm{min}$ at room temperature. The cells were fixed for 5 min with ice-cold fixative (3: 1, methanol: acetic acid). The fixative was changed 3 times. The cells were dropped onto slides which had been cleaned by soaking in $75 \%$ ethanol, and Giemsa-stained. They were analyzed for their chromosome counts, at least 100 metaphase spreads.

\section{Production and analysis of embryoid bodies}

Embryoid bodies were produced by making $20 \mu \mathrm{l}$ of hanging drops containing about 800 cells as described by Rohwedel et al. [12]. In order to induce differentiation into musculature, ESM supplemented with $1 \%$ dimethylsulfoxide (DMSO) was used [10]. After 2 days culture in hanging drops, cell clumps were transferred into a bacteriological petri dish.

These cell clumps were stained for $\beta$-galactosidase activity for 5 days, as described by Yamazaki and Imai
[13]. Five to ten embryoid bodies were picked up, washed with PBS, and then, they were washed 2-3 times with PBS containing $4 \mathrm{mg} / \mathrm{ml}$ polyvinylpyrrolidone. They were fixed with $1.25 \%$ glutaraldehyde in PBS for 5 min. at room temperature, and were washed 3 times with PBS. Then they were transferred into reaction solution (PBS containing 1.2 mM 4-chloro-5-bromo-3indolyl- $\beta$-galactoside (X-gal; WAKO), $0.1 \%$ Triton $\mathrm{X}-100$, $1 \mathrm{mM} \mathrm{MgCl}_{2} \cdot 6 \mathrm{H}_{2} \mathrm{O}, 3 \mathrm{mM} \mathrm{K}$ [ $\left.\mathrm{Fe}(\mathrm{CN})_{6}\right] \cdot 3 \mathrm{H}_{2} \mathrm{O}$ and 3 $\left.\mathrm{mM} \mathrm{K}_{3}\left[\mathrm{Fe}(\mathrm{CN})_{6}\right]\right)$, and incubated for about 24 hours at $37^{\circ} \mathrm{C}$ in a humidified atmosphere.

\section{Results}

\section{Production of transfected sublines}

The survival rate of the cells after electroporation was $61.6 \%$. The thirty sublines which survived after selection with G418 were applied to Southern hybridization using LacZ gene as a probe. The result of the Southern hybridization of 15 sublines (OM1-15) is shown in Fig. 2. It indicates that, of the 30 sublines applied to Southern hybridization, 20 sublines (OM1-6, 8-10, 13, 14, 16, 18, 19, 22, 24, 26-28 and 30) had LacZ gene. There were some lines which had no LacZ gene although it was possible to select them by treatment with G418 (Fig. 2; OM7, 11, 12 and 15).

The data of karyotype analyses of the ESD3 parent cell line and some sublines are shown in Table 1. There were no differences among the karyotypes. We mainly used two sublines, OM9 and OM14, for LacZ gene expression analysis in this study.

\section{Expression of LacZ gene in embryoid bodies}

$X$-gal staining of embryoid bodies indicated that transfected cells did not express in an undifferentiated state and that the number of cells with LacZ expression increased progressively (Fig. 3). In DMSO treated embryoid bodies, the number of cells expressing LacZ gene tended to increase more rapidly than that of untreated them. However, it was unclear whether the cells expressing LacZ gene committed to myogenesis truly.

\section{Discussion}

We attempted to produce a tissue-specific cell marker, especially muscle-specific, by transfecting the transgene, pMGN(-4k)LacZ-neo gene, into ES cells. The survival rate of the cells after electroporation was $61.6 \%$, indicating that conditions used in this study were suitable for transfection of ES cells. Twenty sublines having both LacZ gene and neor gene were obtained, but there 


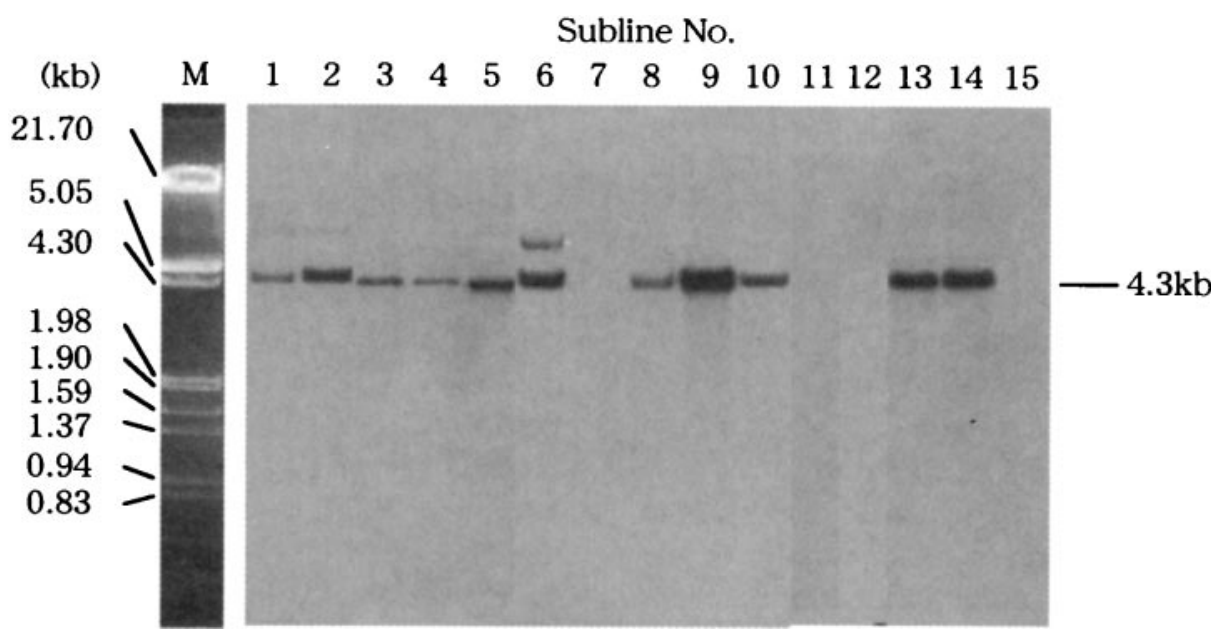

Fig. 2. Results of Southern hybridization of sublines electroporated pMGN(-4k)LacZ-neo gene (OM 1-15). LacZ gene (Bam HI-Bam HI fragment (4.3 kb) from pMGN(-4k)LacZ gene) was used as a probe. There were some lines which had no LacZ gene although they were selected by G418 treatment (OM7, 11, 12 and 15). M: Marker.

Table 1. Karyotype analysis of ESD3 cell line and sublines

\begin{tabular}{lcccccc}
\hline \multirow{2}{*}{ Lines } & \multirow{2}{*}{$\begin{array}{c}\text { No. of cells } \\
\text { counted }\end{array}$} & \multicolumn{5}{c}{ Chromosome counts (\%) } \\
\cline { 3 - 7 } & 102 & 48 & 39 & 40 & 41 & 42 \\
\hline ESD3 & 100 & $6(6.9)$ & $16(15.7)$ & $47(46.1)$ & $29(28.4)$ & $6(5.9)$ \\
OM9 & 100 & $7(7.0)$ & $12(12.0)$ & $47(47.0)$ & $24(24.0)$ & $10(10.0)$ \\
OM14 & 100 &
\end{tabular}

were also some lines which had no LacZ gene although they had neor gene. These lines may be the result of disintegration of the transgene during construction or electroporation, however, we did not carry out groping of the conditions to raise the rate of integration. We also did not examine the number of copies of transgene integrated into the genome of the cells in this study. The results of karyotype analyses indicated no differences among ESD3 parent cells and sublines. Therefore, the nature of sublines after transfection seemed to be based on that of the parent lines.

The pMGN(-4k)LacZ gene used in this study was constructed by Fujisawa et al. [6, 8] to express the LacZ reporter gene at the time of myogenin expression, and its expression in transgenic mice was analyzed by Fujisawa et al. [6] and by Kim et al. [7]. Myogenin is almost specific for skeletal muscle, and is expressed during embryogenesis as early as the first somite/ myotome formation. Kim et al. [7] reported that the myogenin-LacZ gene was expressed at $8.0 \mathrm{dpc}$ in mesodermal tissues of transgenic mouse embryos, suggesting that it may be expressed in the somite prior to myotome formation. Therefore it is potentially involved in the early stage of muscle determination. The expression analysis of the transgene in vitro using two transfected sublines indicated that the cells did not express transgene in an undifferentiated state and that the number of the cells with LacZ gene expression increased progressively (Fig. 3). In DMSO treated embryoid bodies, the number of cells expressing LacZ gene tended to increase more rapidly than that of untreated ones. The results suggest that DMSO may induce mesodermal differentiation of ES cells, and that these cell lines have a possibility to be useful as musclespecific cell markers, although it will be necessary to examine the expression of transgene in chimeric mouse embryos and/or efficacy to analysis of myogenesis in vivo and in vitro.

The sublines obtained in this study may be useful as muscle-specific cell markers for the analysis of early myogenesis since they might express lacZ gene at the time of myogenin expression. However, it will be nec- 
Day $2+0$

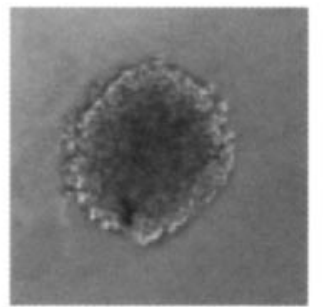

OM 9 (untreated)

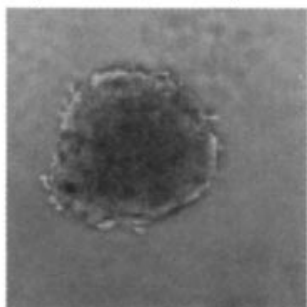

OM 9 (DMSO treated)

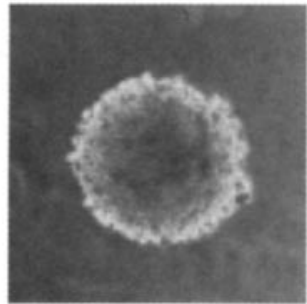

\section{OM14 (untreated)}

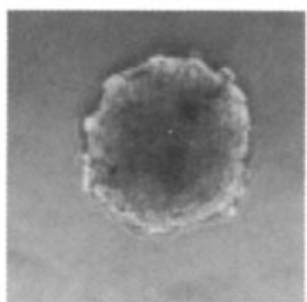

Day $2+1$
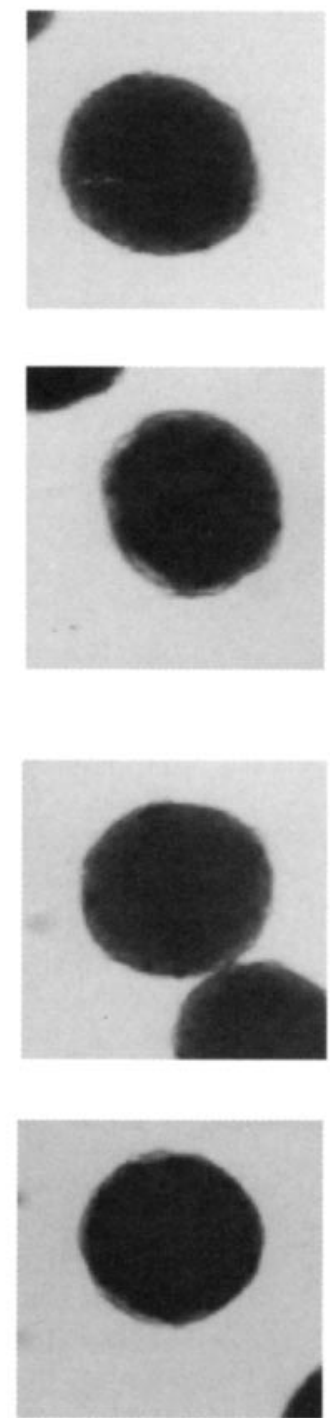

Day $2+3$
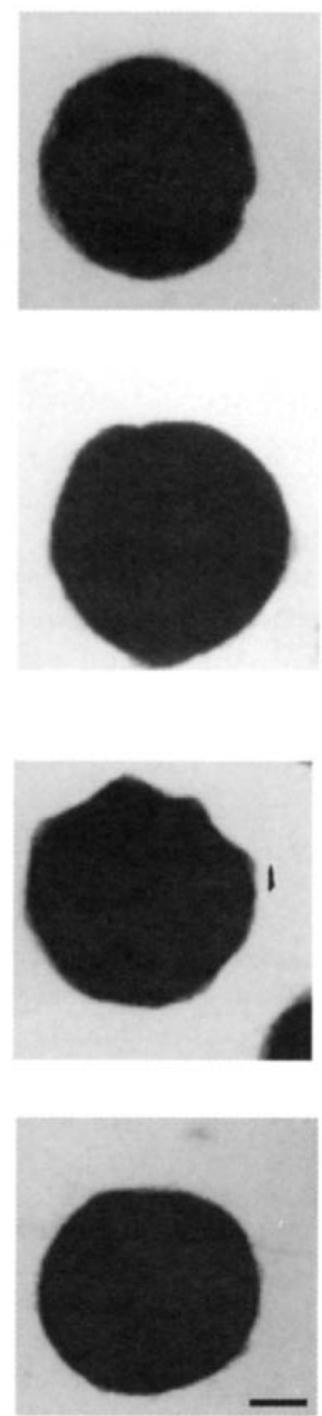

Day $2+3$

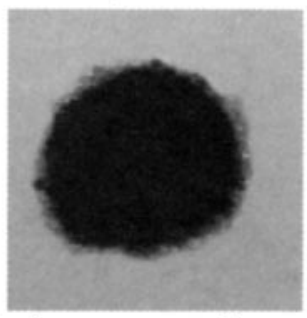

ESD3 (untreated)

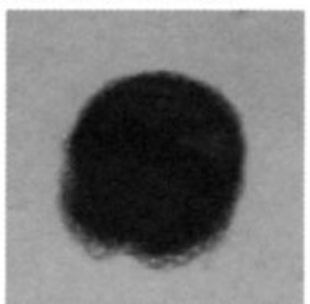

ESD3 (DMSO treated)

\section{OM14 (DMSO treated)}

Fig. 3. Expression of transgene in embryoid bodies derived from OM9 and OM14 untreated or treated with DMSO. The age of embryoid bodies is shown as the sum of days of culture in hanging drop and suspension culture, for example, Day $2+0$ shows the day when they were transferred into bacteriological dishes, and the next day is shown as Day 2 +1 . Transfected cells did not express the transgene in an undifferentiated state and that the number of cells with LacZ expression increased progressively. Although DMSO may induce mesodermal differentiation of ES cells, it is unclear whether the cells expressing LacZ gene committed to myogenesis. Scale bar: $100 \mu \mathrm{m}$.

essary to carry out a detailed analysis of LacZ gene expression in vitro and in chimeric mouse embryos, because there was a possibility of noise with background activity and was a problem of timing of transgene expression. The results in this study shown that, at least, the transgene did not express in an undifferentiated state, but it is necessary to examine the expression of transgene in order to determine the expression pattern of transgene in detail.

From the results of inactivation of myogenin gene, Nabeshima et al. [14] proposed that (1) there may be an intrinsic difference in the muscle differentiation programmes of the two muscle groups, i.e. the muscles derived from myotomal myocites in somites and those 
derived from the cells that migrate from somites, and (2) the growth stimulation to which cells of the two muscle lineage respond may be different. But the molecular function of myogenin in myogenesis remains to be elucidated in detail. It should be possible to use our cells for the analysis of the effects of local environment on muscle differentiation and the function of myogenin in myogenesis of the two muscle groups by locally introducing the cells into postimplantation embryos. Furthermore, the same system may be applicable to studies such as differentiation of other specific tissues or a gene involving diverse functions.

\section{Acknowledgements}

We are grateful to Dr. Atsuko Fujisawa-Sehara, National Institute of Neuroscience, for supplying myogenin-LacZ gene. We are also grateful to Dr. Akiyoshi Fukamizu, Tsukuba university, for supplying a ESD3 cell line.

\section{References}

1) Kusakabe, M., Yokoyama, M., Sakakura, T., N omura, T., Hosick, H. and Nishizuka, Y. (1988): A novel methodology for analysis of cell distribution in chimeric organs using a strain specific antibody. J . Cell Biol., 107, 257-265.

2) Kitani, H., Takagi, N., Atsumi, T., Kawakura, K., Imamura, K., Goto, S., Kusakabe, M. and Fukuta, K. (1996): Isolation of a germline-transmissible embryonic stem (ES) cell line from $\mathrm{C} 3 \mathrm{H} / \mathrm{He}$ mice. Zool. Sci., 13, 865-871.

3) Hanaoka, K., Hayasaka, M., U etsuki, T., FujisawaSehara, A. and Nabeshima, Y. (1991): A stable cellular marker for the analysis of mouse chimeras: the bacterial chloramphenicol acetyltransferase gene driven by the human el ongation factor $1 \alpha$ promoter. Differentiation, 48, 183-189.

4) Kadokawa, Y., Suemori, H. and Nakatsuji, N. (1990): Cell lineage analyses of epithelia and blood vessels in chimeric mouse embryos by use of an embryonic stem cell line expreessing the $\beta$-galactosidase gene. Cell Differ. \& Dev., 29, 187-194.
5) Sassoon, D., Lyons, G., Wright, W., Lin, V., Lassar, A., Weintraub, H. and Buckingham, M. (1989): Expression of two myogenic regulatory factors myogenin and MyoD1 during mouse embryogenesis. Nature, 341, 303-307.

6) Fujisawa-Sehara, A., Hanaoka, K., Hayasaka, M., Hiromasa-Yagami, T. and Nabeshima, Y. (1993): U pstream region of the myogenin gene confers transcriptional activation in muscle cell lineages during mouse embryogenesis. Biochem. \& Biophys. Res. Comm., 191, 351-356.

7) Kim, S.Y., Takahashi, J ., Tsutsumi, K., Kang, M-S. and Yasuda, Y. (1995): Expression of the myogeninLacZ reporter gene during early postimplantation development of the mouse. J. Reprod. Dev., 41, 241247.

8) Fujisawa-Sehara, A., Nabeshima, Y., Hosoda, Y., Obinata, T. and Nabeshima, Y. (1990): Myogenin contains two domains conserved among myogenic factors. J . Biol. Chem., 265, 15219-15223.

9) Doetschman, T.C., Eistetter, H., Katz, M., Schmidt, W. and Kemler, R. (1985): The in vitro development of blastocyst-derived embryonic stem cell lines: formation of visceral yolk sac, blood islands and myocardium. J . Embryol. Exp. Morph., 87, 27-45.

10) Dinsmore, J., Ratliff, J., Deacon, T., Pakzaban, P., J acoby, D., Galpern, W. and I sacson, O. (1996): Embryonic stem cells differentiated in vitro as a novel source of cells for transplantation. Cell Transplantation, 5, 131-143.

11) Robertson, E.J . (1987): E mbryo-derived stem cell lines. In: Teratocarcinomas and Embryonic Stem Cells; A Practical Approach (Robertson E.J . ed.), pp. 71-112, IRL press, Oxford.

12) Rohwedel, J ., Horak, V., Hebrok, M., Fuchtbauer, EM. and Wobus, A. M. (1995): M-twist expression inhibits mouse embryonic stem cell-derived myogenic differentiation in vitro. Exp. Cell Res., 220, 92-100.

13) Yamazaki, Y. and Imai, H. (1992): Differential expression of Bacterial $\beta$-galactosidase gene fused with various promoters in cultured cells and mouse embryos. Anim. Sci. Technol., 63, 601-608, In J apanese.

14) Nabeshima, Y., Hanaoka, K., Hayasaka, M., Esumi, E., Li, S., Nonaka, I. and Nabeshima, Y. (1993): $M$ yogenin gene disruption results in perinatal lethality because of severe muscle defect. Nature, 364, 532535. 